\title{
Análise crítica sobre edulcorantes e flavorizantes empregados na correção sensorial de extratos de Cannabis para uso pediátrico
}

\author{
Critical analysis on sweeteners and flavoring agents used in the sensory \\ correction of Cannabis extracts for pediatric use
}

Recebido em: 05/07/2018

Aceito em: 14/12/2018
Andressa de Souza DUARTE; Carla da Silva CARNEIRO;

Virgínia Martins CARVALHO

Faculdade de Farmácia, Universidade Federal do Rio de Janeiro (UFRJ). Avenida Carlos Chagas Filho, 373, CEP:21941-902. Rio de Janeiro, RJ, Brasil.

E-mail:vmcfarm@gmail.com

\section{ABSTRACT}

Therapeutic treatment with Cannabis extracts is sometimes the only alternative in the control of childhood refractory epilepsy. Cannabis sativa is a banned plant in Brazil, but the importation of cannabis extracts has been authorized for medical use in 2015. However, these formulations are not specific for infant use and compromise compliance with treatment due to palatability. The objective of this study was to propose formulations of cannabis extracts that can serve the child population, considering the incorporation of additives as a flavor masking strategy. For this, the compositions of the extracts available in the market were studied, and searches were made on health databases and regulatory agency websites. It was observed that among the alternatives available on the market, only one was adequate for children's consumption because it incorporated a highly accepted flavoring, besides glycerin, which masks the unpleasant taste of hemp oil. Unconstrained formulations have been suggested for the type of oil incorporated, absent from sweeteners, if the formulation is only oily, and with at least one flavor masking strategy, such as the incorporation of glycerin or the incorporation of flavoring agents. These proposals can be treated with an elaboration of extracts that increase the child adhesion to therapy.

Keywords: cannabidiol; childhood epilepsy; treatment; palatability

\section{RESUMO:}

O tratamento terapêutico com extratos de cannabis representa, por vezes, a única alternativa no controle da epilepsia refratária infantil. Cannabis sativa é uma planta proibida no Brasil, mas a importação de extratos de cannabis foi autorizada para uso médico em 2015. Entretanto, estas formulações não são específicas para uso infantil comprometendo a adesão ao tratamento devido à palatabilidade. Este estudo objetivou realizar uma análise crítica dos edulcorantes e flavorizantes usados na formulações de extratos de cannabis registrados como suplementos alimentares e empregados no Brasil no controle de epilepsia refratária na população pediátrica. Foram estudadas as composições dos extratos disponíveis no mercado e realizadas buscas em bases de dados e sites de agências reguladoras. Dentre as alternativas disponíveis no mercado, apenas uma estava adequada, porque, incorporava um flavorizante de elevada aceitação, além de glicerina, que mascara 
o sabor desagradável do óleo de cânhamo. Foram sugeridas formulações sem restrições ao tipo de óleo incorporado, ausentes de edulcorantes e com pelo menos uma estratégia de mascaramento de sabor, como a incorporação de glicerina ou a incorporação de flavorizantes. Estas propostas podem guiar a elaboração de extratos que aumentem a adesão infantil a terapia.

Palavras-Chave: canabidiol; epilepsia infantil; tratamento; palatabilidade

\section{INTRODUÇÃO}

Os produtos à base de canabidiol disponíveis comercialmente consistem em extratos de Cannabis sativa de variedades denominadas genericamente de cânhamo. O cânhamo inclui plantas do gênero Cannabis ou partes dela, de qualquer variedade, que contêm $0,3 \%$ ou menos de tetrahidrocanabinol (1). No mercado internacional há o óleo de semente de cânhamo que, ao contrário dos extratos oleosos produzidos pela extração de folhas e inflorescências da planta, tem concentrações muito baixas de canabinoides $(1,2)$. Os derivados industriais de cânhamo, tanto o óleo de semente de cânhamo (1), quanto os extratos oleosos preparados a partir da resina de cânhamo, têm diferentes indicações, que incluem suplementação alimentar, uso medicinal e preparação de cosméticos. Dentre as diferentes aplicações terapêuticas que possuem, os extratos de cannabis têm sido alternativa à epilepsia refratária ou fármaco-resistente, que acomete, inclusive, pacientes pediátricos.

Apesar das diferentes indicações de uso dos extratos ricos em canabidiol, o Brasil possui restrições legais ao seu consumo devido ao fato de cannabis ser uma planta proibida pela Convenção Internacional de Drogas, da qual o Brasil é signatário $(3,4)$. A cannabis e seus fitocanabinoides têm seu uso proscrito no Brasil, segundo a Portaria $\mathrm{n}^{\circ} 344$ de 1998, que regula e define os controles e proibições de substâncias no país (5). Entretanto, ocorreram mudanças por meio de publicações mais recentes. A Resolução da Diretoria Colegiada (RDC) $n^{\circ} 3$ de 2015 da Agência Nacional de Vigilância Sanitária (Anvisa) atualizou a lista $\mathrm{C} 1 \mathrm{da}$ portaria anteriormente mencionada de maneira a incluir o canabidiol (6) como fármaco sob controle especial que pode ser prescrito. Em seguida, a RDC $\mathrm{n}^{\circ} 17$ de 2015 autorizou a importação de produtos à base de canabidiol sem registro no país, em caráter de excepcionalidade e para uso terapêutico. A RDC também apontou os critérios e procedimentos da importação dos produtos definindo que se trata de produto específico sem comprovação de eficácia e segurança (7).

Estudos têm mostradoevidências da eficácia do uso de canabidiol no tratamento de diversos tipos de doenças, tais como epilepsia refratária $(8,9)$, esquizofrenia (10), ansiedade (11) ou epilepsia $(10,11)$. As alterações observadas recentemente na legislação brasileira, e citadas no parágrafo anterior, foram impulsionadas pela persistência de grupos de mães cujos filhos têm epilepsia refratária e ativistas. Sendo assim, atualmente, e com a autorização da Anvisa, os produtos à base de canabidiol importados ao Brasil para uso terapêutico podem ser comprados principalmente dos Estados Unidos da América, que os comercializa originalmente como suplemento alimentar.

No caso da epilepsia refratária, muitos pais têm recorrido à alternativa da administração via oral de extratos à base de canabidiol, pois tem se demonstrado eficaz na redução da frequência de crises convulsivas quando terapias convencionais não funcionam $(8,9)$. Entretanto, para que o tratamento seja eficiente aos pacientes pediátricos, é importante que, para este grupo específico, o desenvolvimento da formulação do extrato considere aspectos diferentes em relação à produção convencional. Isso ocorre pois crianças são diferentes de adultos e demonstram características peculiares que podem comprometer a adesão à terapia caso a formulação seja a mesma $(12,13)$. Apesar da conhecida necessidade de adaptar formulações de uso terapêutico para a administração em pediátricos, não existem alternativas disponíveis de extratos ricos em canabidiol voltados especificamente ao tratamento de pacientes em idade infantil.

Quanto às características comuns da composição dos extratos disponíveis atualmente no mer- 
cado, além de não serem específicos para uso pediátrico, quase todos os produtos não apresentam edulcorantes, que contribuem na melhoria da palatabilidade. Entretanto, parte das empresas fornecem alternativas contendo flavorizantes, o que pode ser a opção mais proveitosa ao uso infantil. Quanto a outros aditivos da composição, alguns produtos apresentam vários tipos de óleos diferentes do óleo de semente de cânhamo. Por último, alguns produtos apresentam em sua composição glicerina e propilenoglicol como diluente, outros contem antioxidantes ou polissorbato como tensoativo.

Com base nos dados expostos sobre o tratamento de epilepsia refratária infantil e também baseado na composição dos extratos de cannabis já disponíveis para o uso terapêutico, o presente trabalho teve como objetivo propor, por meio de uma análise crítica, formulações de extratos de cannabis, para a administração oral, que possam atender à população infantil, considerando a incorporação de aditivos como estratégia de mascaramento de sabor.

\section{MÉTODOS}

Tipo de estudo e fonte de dados. $\mathrm{O}$ trabalho foi elaborado a partir de uma revisão bibliográfica utilizando como ferramenta as bases de dados " $\mathrm{Pu}$ bmed', "SciELO", Google Acadêmico e "Toxicology Data Network" (TOXNET). Foram utilizados os descritores, "cannabinoid for epilepsy", "children medication", "children palatability", "flavored medication", "artificial sweeteners". Agências reguladoras como a Agência Nacional de Vigilância Sanitária (Anvisa), "Food and Drug Administration" (FDA) e "Health Canada" também foram consultadas. Algumas informações também foram obtidas de empresas fornecedoras de produtos que contêm canabidiol.

Critérios de inclusão e de exclusão. Quanto aos critérios de exclusão da pesquisa, foram desconsiderados trabalhos que especificavam o consumo de canabinoides ou de qualquer outro possível componente da formulação por vias diferentes da via oral. Também foram excluídos trabalhos que não tinham como objeto de estudo extratos de natureza oleosa. Por outro lado, tanto para a identificação quanto para a proposição da formulação mais adequada para uso pediátrico, foram considerados os componentes que promoveriam o melhor perfil de adesão e os impactos positivos à saúde associados ao consumo contínuo. Por último, os dados considerados para este trabalho foram coletados entre os anos de 1964 e 2018.

\section{RESULTADOS E DISCUSSÃO}

Levantamento de extratos de cannabis. A Tabela 1 apresenta resultados do levantamento de extratos de cannabis disponíveis no mercado no período de 2016 a 2018. Poucas formulações possuíam o edulcorante estévia e algumas continham flavorizantes. Por último, algumas formulações não se apresentaram exclusivamente oleosas, pois continham glicerina, incorporada em algumas formulações com polissorbato.

Palatabilidade infantil $x$ aceitação de extratos de cannabis. A palatabilidade infantil é um aspecto importante de ser analisado para a proposição da formulação, pois crianças possuem percepções e preferências distintas de sabor em relação aos adultos. Essas percepções são modificadas com o tempo por meio de experiências (14) e devido ao fato de sensibilidade aos sabores sofrer variações ao longo da infância(15). Em função disso, existem desafios extras na administração de formas farmacêuticas para pacientes pediátricos e, no caso dos extratos oleosos de cannabis, existe o empecilho adicional de possuírem sabor não atrativo, o que pode ser considerado uma desvantagem, pois há prejuízos na adesão à terapia.

Associado às diferenças de percepção infantil dos sabores, a revisão de estudos experimentais permitiu observar que, comparativamente a adultos, crianças apresentam maior apreciação pelo sabor doce $(16,17)$ e maior rejeição pelo sabor amargo $(16,18,19)$. Os estudos comparativos também mostraram que crianças têm menor preferência por alimentos oleosos/gordurosos (20) e maior apreciação pelo sabor ácido $(18,21)$. Quanto aos estudos não comparativos com adultos, foi observado que crianças apreciam o sabor salgado $(14,22)$. Além disso, em um estudo de mascaramento de sabor amargo da prednisona, foi demonstrado que a incorporação de sabores exclusivamente salgados ou 
exclusivamente ácidos não promoveu aumento relevante da aceitação infantil, enquanto a associação de qualquer um desses sabores com o sabor doce promoveu a aceitação máxima (23).

Além da percepção geral dos sabores pela população infantil, alguns estudos fizeram apontamentos em relação aos sabores de flavorizantes considerados os de maior aceitação infantil. Dessa forma, baseado na produção industrial de doces e em ordem de preferência, estão: tutti-frutti, morango, cereja, melancia, laranja, framboesa azul, sabores ácidos, uva e maçã verde (24). Além disso, a preferência infantil apresenta resultados diferentes quando se analisa apenas o conjunto dos países da América do Sul. Nesse caso, a ordem de preferên- cia torna-se: morango, laranja, tutti-frutti, abacaxi, uva, maçã verde, manga, limão, pêssego, tamarindo e cereja (25).

Restrições alimentares comuns na epilepsia refratária. Para a proposição da formulação, também devem ser consideradas as restrições alimentares comuns na epilepsia refratária. Devido a isso, é importante observar que parte dos pacientes pediátricos que fazem tratamento com extratos de cannabis utilizam simultaneamente a dietacetogênica. A dieta cetogênica consiste na ingestão reduzida de carboidratos, adequada de proteínas, consequentemente, mais elevada de lipídios $(26,27)$ e é eficaz em alguns casos no controle de crises convulsivas, principalmente na população infantil $(28,29)$.

Tabela 1. Síntese do levantamento de extratos de cannabis disponíveis no mercado (Período 2016-2018)

\begin{tabular}{|c|c|c|c|}
\hline Produto & Fase oleosa & Edulcorante & Flavorizante \\
\hline P1 & óleo de cânhamo(CBD), óleo de coco (“MCT oil”), vitamina E. & ND & ND \\
\hline P2 & Óleo de cânhamo ultra refinado, glicerina, Polissorbato 80 & ND & ND \\
\hline P3 & Óleo de cânhamo(CBD), óleo de coco ("MCT oil”), vitamina E & ND & ND \\
\hline P4 & $\begin{array}{l}\text { Óleo de cânhamo, óleo de cânhamo virgem e orgânico (extraído das } \\
\text { sementes), vitamina E. }\end{array}$ & ND & ND \\
\hline P5 & Glicerina, Óleo de cânhamo(CBD) & ND & Flavorizante natural de morango \\
\hline P6 & Glicerina, Óleo de cânhamo(CBD) & ND & Flavorizante natural de blueberry \\
\hline P7 & Glicerina, Óleo de cânhamo(CBD) & ND & ND \\
\hline P8 & Glicerina, Óleo de cânhamo(CBD) & ND & Flavorizante natural de maçã verde \\
\hline P9 & Glicerina, Óleo de cânhamo(CBD) & ND & Flavorizante natural de canela \\
\hline P10 & óleo de coco («MCT oil»), Óleo de cânhamo(CBD) & ND & ND \\
\hline P11 & $\begin{array}{l}\text { Óleo de cânhamo bruto, Óleo de cânhamo (extraído das sementes), óleo } \\
\text { de semente de uva, Terpenos }\end{array}$ & ND & Flavorizantes alimentares \\
\hline P12 & $\begin{array}{l}\text { Canabidiol (CBD) de extrato oleoso de cânhamo, óleo de olíbano (Boswellia } \\
\text { carteri), óleo de semente de cominho preto (Nigella sativa), extrato de } \\
\text { alecrim (conservante natural) }\end{array}$ & ND & ND \\
\hline $\mathrm{P} 13$ & Extrato de cânhamo (partes aéreas), óleo de coco fracionado ("MCT oil") & ND & $\begin{array}{l}\text { Óleo flavorizante sabor chocolate } \\
\text { e hortelã, flavorizantes naturais e } \\
\text { orgânicos }\end{array}$ \\
\hline P14 & Extrato de cânhamo (partes aéreas), óleo de oliva orgânico extra virgem & ND & ND \\
\hline P15 & $\begin{array}{l}\text { Óleo de cânhamo (extraído com CO2), óleo de semente de cânhamo, óleo } \\
\text { de coco }\end{array}$ & Estévia & ND \\
\hline P16 & $\begin{array}{l}\text { Óleo de cânhamo, óleo de semente de cânhamo, óleo de semente de uva, } \\
\text { glicerina }\end{array}$ & Estévia & Extrato de baunilha \\
\hline
\end{tabular}


Devido à possibilidade de o paciente em tratamento com extratos de cannabis também fazer dieta cetogênica, é importante que as formulações voltadas ao uso infantil não possuam componentes que alterem a glicemia e, assim, não comprometam o estado de cetose. Uma estratégia viável de avaliação da adequação dos componentes à formulação seria a análise de seus respectivos índices glicêmi$\cos$ (IG). O IG corresponde ao efeito na glicemia produzido por carboidratos comparativamente ao efeito de uma quantidade igual de glicose, sendo, para isso, estabelecida uma relação (30). Para a glicose é considerado $\mathrm{IG}=100$ (30) e, no caso dos componentes selecionáveis à formulação, é importante que tenham IG igual a zero.

Para melhorar a adesão ao tratamento de epilepsia refratária infantil uma das estratégias aplicáveis à proposta de formulação consiste na incorporação de aditivos que possam conferir sabor doce ao extrato, como os edulcorantes. No Brasil, segundo a RDC n ${ }^{\circ} 18$, de 24 de março de 2008 (31), são 15 os edulcorantes autorizados pela Anvisa, alguns naturais e outros artificiais (Tabela 2).

Tabela 2. Propriedades dos edulcorantes avaliados para formulações contendo extrato de cannabis

\begin{tabular}{|c|c|c|c|c|c|c|}
\hline & $\begin{array}{l}\text { Índice } \\
\text { Glicêmico }\end{array}$ & Resultado & $\begin{array}{c}\text { Sabor } \\
\text { Amargo }\end{array}$ & Resultado & $\begin{array}{c}\text { Sabor } \\
\text { metálico }\end{array}$ & Resultado \\
\hline Acessulfame de potássio & 0 & & Sim & -- & Sim & -- \\
\hline ácido ciclâmico (sais de) & 0 & & Sim & -- & Sim & -- \\
\hline Aspartame & 0 & & Sim & -- & Sim & -- \\
\hline Eritrol & 0 & & Não & & Não & \\
\hline Estévia & 0 & & Sim & -- & Não & \\
\hline Lactitol & 6 & -- & Não & & Não & \\
\hline Isomaltitol & 9 & -- & Não & & Não & \\
\hline Maltitol & 35 & -- & Não & & Não & \\
\hline Manitol & 0 & & Não & & Não & \\
\hline Neotame & 0 & & Não & & Não & \\
\hline Sacarina (sais de) & 0 & & $\operatorname{sim}$ & -- & Sim & -- \\
\hline Sucralose & 0 & & Não & & Não & \\
\hline Sorbitol & 9 & -- & Não & & Não & \\
\hline Taumatina & 4 & -- & Não & & Não & \\
\hline Xilitol & $8-13$ & -- & Não & & Não & \\
\hline
\end{tabular}

Adaptado de Grembecka (2005) (32), American Dietetic Association (2004) (33), Riera e cols.., 2007 (34) e Singla e cols., 2016 (35). - -: Edulcorantes considerados inadequados para a proposta.

Entretanto, considerando o que foi anteriormente abordado, para que os edulcorantes possam ser apontados como boas alternativas à formulação, é importante que não alterem a glicemia, a fim de preservar o estado de cetose; e também tenham sabor adequado à palatabilidade infantil, para melhorar a adesão. Em função disso, podem ser utilizados como critério de exclusão edulcorantes com índice glicêmico (IG) diferente de zero ou com retrogosto amargo ou metálico (Tabela 2). Como pode ser observado naTabela 2 e considerando os critérios de exclusão, dos 15 edulcorantes autorizados para consumo no Brasil, apenas quatro seriam selecionáveis à proposta: manitol, eritrol, sucralose e neotame.

Flavorizantes. Outra alternativa para aumentar a adesão à terapia seria a incorporação de flavorizantes à formulação dos extratos de cannabis. Com base nisso, alguns autores afirmaram que é possível associar o uso de tipos específicos de flavorizantes 
com o sabor que se deseja reprimir $(36,37)$ (Quadro 1). Podem ser identificadas as alternativas de flavorizantes mais adequadas para mascarar especificamente os sabores oleoso e amargo, presentes na formulação. Entretanto, considerando o que já foi pontuado a respeito da palatabilidade infantil e de suas preferências de sabores, convém que, dentre os flavorizantes indicados para o mascaramento de ambos os sabores, seja dada a preferência aos que também tem maior aceitação pela população infantil. Assim, para a incorporação ao extrato de cannabis, seria preferível o uso dos flavorizantes limão, laranja, cereja e framboesa, pois teriam elevada aceitação e mascarariam o sabor amargo simultaneamente.

Quadro 1. Flavorizantes adequados para mascarar/ disfarçar sabores

\begin{tabular}{|c|c|c|}
\hline Sabor & Ferreira (2002) & $\begin{array}{c}\text { Allen e cols } \\
\text { (2013) }\end{array}$ \\
\hline Amargo & $\begin{array}{c}\text { chocolate, chocolate e } \\
\text { menta, menta, limão, } \\
\text { laranja, cereja, framboesa }\end{array}$ & Coco \\
\hline Oleoso & menta, canela & - \\
\hline
\end{tabular}

Adaptado de Ferreira (2002)(36) e Allen e cols., (2013) (37).
Além da adequação entre o flavorizante e o sabor que se deseja mascarar, existem diferenças quanto ao critério de quantificação para a posterior incorporação na formulação proposta. Assim, para o cálculo da quantidade adequada do flavorizante a ser adicionado, há um procedimento padronizado de acordo com a natureza físico-química ou estado físico do flavorizante usado (37) (Quadro 2).

Óleos e glicerina. As formulações de extratos de cannabis disponíveis comercialmente possuem como componente principal a resina oleosa do cânhamo, onde se concentram os canabinoides com ação terapêutica de interesse. Além disso a resina contendo canabinoides é solubilizada em diferentes tipos de óleo, como o óleo de semente de cânhamo e outros óleos que, normalmente, atuam como diluentes e não possuem ação direta na melhora da palatabilidade. Assim, é importante que também sejam analisadas as propriedades associadas a cada tipo de óleo e seus possíveis impactos na saúde. Para a análise, foram considerados todos os tipos de óleos identificados nas formulações disponíveis no mercado, mais a glicerina, que também estava presente em alguns produtos (Tabela 1).

Quadro 2. Planejamento da introdução de flavorizantes em formulações contendo extratos de cannabis

\begin{tabular}{|l|l|}
\multicolumn{1}{c|}{ Tipo de flavorizante } & \multicolumn{1}{c}{ Concentração adequada } \\
\hline Flavorizantes solúveis em água & $\begin{array}{l}\text { Iniciar com a concentração de } 0,2 \% \text { para os flavorizantes artificiais e de } 1 \text { a } 2 \% \text { para os } \\
\text { naturais }\end{array}$ \\
\hline Flavorizantes lipossolúveis & $\begin{array}{l}\text { Iniciar com a concentração de } 0,1 \% \text { para os flavorizantes artificiais e } 0,2 \% \text { para os na- } \\
\text { turais em produtos acabados }\end{array}$ \\
\hline Flavorizantes em pó & $\begin{array}{l}\text { Iniciar com a concentração de } 0,1 \% \text { para os flavorizantes artificiais e } 0,75 \% \text { para os } \\
\text { naturais nos produtos acabados }\end{array}$ \\
\hline
\end{tabular}

Adaptado de Allen e cols. (2013) (37).

Propriedades do óleo de semente de cânhamo. O componente principal das formulações apresenta sabor amargo (38) e suas propriedades descritas por estudos clínicos estão associadas principalmente ao sistema nervoso central. Os estudos clínicos descrevem ações anticonvulsivante/ antiepiléptica $(39,40)$, ansiolítica (11) e antipsicótica para pacientes com esquizofrenia (41). Também são apontadas ações anticarcinogênica em pacientes com anemia linfoblástica aguda (38).

\section{Propriedades dos outros óleos presentes nas} formulações. Além do óleo de cânhamo, as formulações comercializadas continham ao todo outros sete óleos. No Quadro 3 é apresentado o levantamento dos óleos presentes nas formulações e as suas respectivas propriedades terapêuticas descritas por estudos clínicos.

Critérios para a seleção de óleos diluentes. O estudo pontual dos sete óleos diluentes mostrou aplicações terapêuticas diferentes das comumente 
demandadas pela população de pacientes em tratamento de epilepsia refratária. Isso pode ser apontado como justificativa para o não estabelecimento de restrições à incorporação de qualquer um dos óleos diluentes à proposta de extrato de cannabis para uso pediátrico. Entretanto, é possível que sejam estabelecidas preferências na seleção de óleos de acordo com as particularidades de cada paciente. Por exemplo, ao óleo de semente de cominho preto poderia ser dada maior preferência de uso para pacientes com histórico de reações alérgicas ou com tendência à lesão hepática.

Propriedades da glicerina. Além dos óleos, algumas formulações também continham glicerina, estabilizada ou não com polissorbato 80 . A glicerina é um álcool do tipo poliol, solúvel em água e etanol e que possui funções farmacotécnicas de umectante e solvente (52). No caso das formula- ções com glicerina, além de o componente atuar como solvente, sua incorporação também se aplica como uma estratégia peculiar no mascaramento do sabor desagradável do óleo de cânhamo, pois, por ser um poliol, a glicerina possui sabor levemente adocicado (53). Além disso, a presença do polissorbato 80 permite a formação de uma emulsão que reduziria ainda mais o contato da fase oleosa com as papilas gustativas. $\mathrm{O}$ polissorbato 80 tem ação solubilizante, emulsionante e umectante (52).

A glicerina é bastante descrita na literatura como excipiente de preparações farmacoténicas, (Tabela 1). Entretanto, quanto a suas aplicações clínicas, são apontados o uso em enemas e supósitórios no tratamento de constipação $(54,55)$ e, quanto ao uso oral, estudos antigos apontavam seu uso na redução da pressão ocular na pré-cirurgia oftalmológica ou no tratamento de glaucoma $(56,57)$.

Quadro 3. Propriedades terapêuticas dos óleos, descritas por estudos clínicos

\begin{tabular}{|c|c|}
\hline Óleo & Propriedades descritas \\
\hline Óleo de coco & $\begin{array}{l}\text { Redução dos niveis de LDL e colesterol total em comparação ao consumo de gorduras } \\
\text { saturadas, como manteiga, gordura animal, óleo de palma }(42,43) \\
\text { Aumento dos níveis de LDL e colesterol total em comparação ao consumo de gorduras } \\
\text { insaturadas, como óleo de milho e óleo de oliva (44) }\end{array}$ \\
\hline Óleo de semente de uva & $\begin{array}{l}\text { Redução da incidência de câncer de próstata em } 41 \%(45) \\
\text { Elevação dos níveis de } \mathrm{HDL} \text { e redução dos níveis de LDL associados ao consumo } \\
\text { experimental de } 45 \mathrm{~g} / \text { dia (46) }\end{array}$ \\
\hline Óleo de semente de cominho preto & $\begin{array}{l}\text { Ação antihistamínica: redução de IgE e contagem de eosinófilos em pacientes com } \\
\text { doenças alérgicas ( } 47) \\
\text { Ação hepatoprotetora: pacientes com hepatite C; pacientes com leucemia linfoblática } \\
\text { aguda tratadas com metrotrexato (48) }\end{array}$ \\
\hline Extrato oleoso de alecrim & \multirow{2}{*}{ Não foram encontrados estudos clínicos } \\
\hline Óleo de Olíbano ou Frakincense & \\
\hline Óleo de oliva orgânico extravirgem & $\begin{array}{l}\text { Redução da pressão arterial (49) } \\
\text { Prevenção de diabetes melitus tipo II (50) } \\
\text { Redução da incidência de doenças cardiovasculares (51) }\end{array}$ \\
\hline
\end{tabular}

Associação de componentes do veículo a efeitos laxativos. A análise do risco de efeitos laxativos associados ao consumo dos óleos ou glicerina se justifica porque, dentre as estratégias terapêuticas para a indução de efeitos laxativos, são utilizados agentes lubrificantes, como o óleo mineral, óleo de rícino ou parafina (58). Entretanto, não foram encontrados estudos que descrevessem pontualmente os óleos (Tabela 1) como agentes laxativos. Quanto à glicerina, a literatura fornece dados que descrevem seu uso como laxante, mas não por via oral, como ocorre com os produtos listados $(54,55)$. Dessa forma, não são esperados efeitos laxativos associados à administração oral de extratos contendo qualquer um dos componentes mencionados.

Compatibilidade farmacotécnica. Outro aspecto importante a ser considerado na proposta de formulação de extrato de cannabis voltado ao uso infantil é a compatibilidade farmacotécnica dos 
componentes ativos e óleos com os aditivos considerados, no caso, os edulcorantes e flavorizantes. Para isso é importante analisar dois aspectos relacionados: solubilidade e viscosidade.

Solubilidade dos aditivos. Quanto a esse aspecto, é importante que sejam selecionados flavorizantes ou edulcorantes solúveis no veículo, pois, dessa forma, ocorre aumento do contato do aditivo com as papilas gustativas e consequentemente o realce do sabor (36). Ao contrário, a baixa solubilização seria uma estratégia indesejada de redução da percepção $(36,37)$. Com base nisso, para a incorporação na fase oleosa é importante que os aditivos sejam lipofílicos. A exceção a ser considerada seriam as formulações com glicerina, pois sua fase, que teria mais contato com as papilas gustativas, poderia ser incorporada com aditivos hidrofílicos que com ela se solubilizariam.

Com base nesse critério, é importante que sejam observadas as respectivas solubilidades dos edulcorantes considerados selecionáveis (Quadro 4). Com base nos dados observados de solubilidade, os quatro edulcorantes selecionáveis possuem natureza hidrofílica, o que dificulta a incorporação na fase oleosa. Já para formulações com emulsão de glicerina, o manitol e a sucralose, nesta ordem, seriam opções viáveis, uma vez que, segundo a referência, se solubilizariam em glicerina e álcool, respectivamente.

Quadro 4. Solubilidade dos edulcorantes selecionáveis à proposta de formulações contendo extratos de cannabis

\begin{tabular}{|l|l|}
\hline Edulcorante & \multicolumn{1}{c|}{ Solubilidade } \\
\hline Manitol & Solúvel em água e glicerol \\
\hline Eritrol & Muito solúvel em água, pouco solúvel em álcool e insolúvel em benzeno \\
\hline Sucralose & Solúvel em álcool e não solúvel em lipídios \\
\hline Neotame & Solúvel em água \\
\hline
\end{tabular}

Adaptado de PUBCHEM (2017) (59), Mercola e cols. (2006) (60)

Viscosidade do veículo. Além da solubilidade, a viscosidade do veículo também é um parâmetro relevante, pois quanto maior, menor a percepção do sabor do que está solubilizado. Isso ocorre porque, apesar de ter ocorrido a solubilização, o contato dos componentes com as papilas gustativas fica reduzido (36). Dessa maneira, um óleo diluente de alta viscosidade, por exemplo, reduziria a percepção do sabor desagradável do óleo de cânhamo e do seu próprio sabor. Em contrapartida, a incorporação de edulcorantes e flavorizantes a esse óleo, ainda que sejam lipofílicos, também reduziria consideravelmente a capacidade dos aditivos de melhorar o sabor por mascaramento.

\section{Identificação e proposta de formulação de} extrato de cannabis. Para a seleção e proposta de formulações para o uso infantil, foram considerados os parâmetros analisados neste trabalho cujos resultados obtidos apontam as características consideradas ideais ou não de serem aplicadas ao extrato de cannabis para uso infantil (Quadro 5).
Considerando os parâmetros apontados no Quadro 5, para que a formulação seja considerada ideal para uso infantil, foram observados os critérios que devem ser seguidos para a proposição (Quadro 6).

Com base nas informações que foram detalhadas e no levantamento de produtos (Tabela 1), a formulação presente no mercado que mais se adequa a proposta para o aumento da adesão infantil à terapia seria o produto $5(\mathrm{P} 5)$, que é composto de glicerina, óleo de cânhamo(CBD) e flavorizante natural de morango.

O P5 possui como aspectos positivos ser flavorizado com sabor morango, flavorizante considerado de elevada preferência infantil; composto de óleo de cânhamo com glicerina, que tem papel no mascaramento do sabor amargo do óleo. E, como aspecto negativo, ressaltasse o fato de a formulação não conter nenhum agente solubilizante/emulsionante, que melhoraria a solubilidade e homogeneização dos componentes, e, consequentemente, 
o poder flavorizante do incorporado. Além do produto descrito acima (P5), outras duas formulações presentes no mercado possuem constituição que se aproximam a proposta de adequação ao público infantil. São eles o produto 2 (P2) e o produto 13 (P13) (Tabela 1).

Quadro 5. Informações relevantes à formulação infantil contendo extrato de cannabis

\begin{tabular}{|c|c|}
\hline Parâmetro analisado & Informações relevantes à formulação \\
\hline latabilidade infantil & $\begin{array}{l}\text { Crianças preferem o sabor doce, mas também apreciam a associação como sabor ácido suave e } \\
\text { salgado; } \\
\text { Crianças rejeitam o sabor amargo; } \\
\text { A ordem de preferência infantil de sabores é: } \\
\text { Tutti-frutti, morango, cereja, melancia, laranja, framboesa azul, sabores ácidos, uva e maça verde; } \\
\text { A ordem de preferência infantil de sabores, considerando a população da América do Sul é: } \\
\text { morango, laranja, tutti frutti, abacaxi, uva, maça verde, manga, limão, pêssego, tamarindo e cereja }\end{array}$ \\
\hline Restrição alimentar & $\begin{array}{l}\text { Parte dos pacientes com epilepsia fazem dieta cetogênica como estratégia terapêutica. Logo, devem } \\
\text { ser excluídos os aditivos que alteram a glicemia. }\end{array}$ \\
\hline Edulcorantes & $\begin{array}{l}\text { Dos } 15 \text { edulcorantes permitidos no Brasil, apenas manitol, eritrol, sucralose e neotame tem índice } \\
\text { glicêmico igual a zero e não produzem retrogosto amargo ou metálico. }\end{array}$ \\
\hline Flavorizantes & $\begin{array}{l}\text { Os sabores ideias para mascarar sabor oleoso são: Menta e canela } \\
\text { Os sabores ideais para mascarar o sabor amargo são: chocolate, chocolate + menta, menta, limão, } \\
\text { laranja, cereja, framboesa }\end{array}$ \\
\hline óleos & $\begin{array}{l}\text { O óleo de cânhamo tem sabor amargo } \\
\text { Não foi possível estabelecer preferências de uso para os óleos }\end{array}$ \\
\hline $\begin{array}{l}\text { Compatibilidade } \\
\text { Farmacotécnica }\end{array}$ & $\begin{array}{l}\text { Os } 4 \text { edulcorantes selecionáveis são hidrofilicos e não podem ser solubilizados em formulações } \\
\text { apenas oleosas } \\
\text { Manitol e sucralose tem boa solubilidade em glicerina } \\
\text { Partículas reduzidas de aditivo melhoram sua solubilidade } \\
\text { A viscosidade reduzida do veículo melhora a percepção do sabor dos aditivos }\end{array}$ \\
\hline
\end{tabular}

O P2 é composto de óleo de cânhamo ultra-refinado, glicerina vegetal orgânica, Polissorbato 80 . Possui como aspecto positivo ser composto de óleo de cânhamo com glicerina e tensoativo. A glicerina tem sabor adocicado e reprime o sabor desagradável do óleo de cânhamo, e como aspecto negativo, não ser flavorizado. A incorporação de um flavorizante hidrofílico adequado a preferência infantil melhoraria a adesão.

O P13 é composto de extrato de cânhamo (partes aéreas), óleo de coco fracionado (triglicerídeo de cadeia média), flavorizante sabor chocolate e hortelã, flavorizantes naturais e orgânicos. Como aspecto positivo destaca-se a flavorização com os sabores menta e chocolate, que são eficazes no mascaramento, tanto do sabor oleoso, quanto do sabor amargo presentes na formulação, que é exclusivamente oleosa. Como aspecto negativo, o sabor de menta com chocolate não está entre os preferidos pela população infantil, logo seria uma barreira a adesão.

Entretanto, apesar de ser possível selecionar um produto já disponível no mercado cuja formulação esteja mais ajustada à ideal, os três produtos sugeridos apresentam características que precisariam ser modificadas para serem consideradas de total adequação. Dessa forma, alguns exemplos de novas formulações propostas para melhorar a adesão infantil à terapia são descritos a seguir. 
Quadro 6. Critérios para a seleção e proposição da formulação de extrato de cannabis para uso infantil

\begin{tabular}{|c|c|}
\hline Componentes & Considerações \\
\hline Veículo (óleos e glicerina) & $\begin{array}{l}\text { Pode ser constituído de: } \\
\text { Óleo de cânhamo + qualquer outro óleo listado (não foram encontradas informações que } \\
\text { justificassem alguma restrição, mas podem ser analisados os critérios de preferência) } \\
\text { Observação: incorporar flavorizantes lipofílicos por necessidade de mascarar o sabor } \\
\text { desagradável } \\
\text { Óleo de cânhamo + glicerina + agente emulsionante } \\
\text { Observação: a glicerina mascararia o sabor desagradável, mas podem ser adicionados } \\
\text { flavorizantes hidrofilicos }\end{array}$ \\
\hline Flavorizantes & $\begin{array}{l}\text { Para formulações apenas oleosas: optar por laranja, cereja, framboesa e limão } \\
\text { Justificativa: mascaram sabor amargo e são adequados à preferência infantil } \\
\text { Para formulações com glicerina: incorporar qualquer um dos flavorizantes de preferência } \\
\text { infantil, além dos anteriores (ex: tutti-frutti, morango, uva) } \\
\text { Justificativa: a glicerina também cumpriria o papel de reprimir o sabor amargo da fase oleosa }\end{array}$ \\
\hline Edulcorantes & $\begin{array}{l}\text { Para formulações apenas oleosas: não incorporar edulcorantes } \\
\text { Justificativa: os } 4 \text { selecionáveis são hidrofilicos } \\
\text { Para formulações com glicerina: incorporar preferencialmente a sucralose, ou o manitol } \\
\text { Justificativa: ambos teriam a melhor solubilidade, mas a sucralose tem maior poder adoçante } \\
\text { e poderia ser incorporado em menor quantidade }\end{array}$ \\
\hline
\end{tabular}

\section{Proposição de adequação para formulações}

Formulações exclusivamente oleosas. Para formulações exclusivamente oleosas, considerando qualquer um dos óleos listados (Tabela 1), sugere-se restringir o uso de flavorizantes aos sabores laranja, cereja, framboesa e limão e não incorporar edulcorantes. Proposição de formulação oleosa 1: óleo de cânhamo, óleo de oliva extra virgem, flavorizante sabor laranja.

Proposição de formulação oleosa 2: óleo de cânhamo, óleo de semente de uva, flavorizante sabor framboesa.

Formulações com glicerina. Para formulações com glicerina sugere-se incorporar tensoativos ou emulsionantes para promover a interação álcool-óleo e, consequentemente, o mascaramento adequado do sabor; utilizar qualquer um dos flavorizantes que sejam adequados à preferência infantil ou incorporar edulcorante sucralose.

Proposição de formulação com glicerina 1 : óleo de cânhamo, glicerina, polissorbato 80 , flavorizante sabor tutti-frutti.

Proposição de formulação com glicerina 2: óleo de cânhamo, glicerina, polissorbato 80 , flavorizante sabor macã verde, sucralose.

\section{CONCLUSÃO}

As propostas sugeridas podem guiar a elaboração de extratos que aumentem a adesão infantil à terapia, pois ocorreria um mascaramento adequado dos sabores desagradáveis presentes no extrato oleoso de cannabis, juntamente com a percepção de sabores que seriam do agrado da população infantil. Entretanto, apesar de ser possível importar extratos de cannabis para uso terapêutico, e, por meio deste trabalho, conhecer estratégias sensoriais para que sejam selecionados produtos mais palatáveis para administração oral em crianças, a atual limitação da produção nacional de extratos de cannabis para a terapia de epilepsia refratária coopera, na prática, para a restrição da produção de extratos específicos para a terapia infantil, dificultando o acesso à terapia adequada para essa população.

\section{AGRADECIMENTOS}

Os autores agradecem à equipe do projeto de extensão universitária Farmacannabis-UFRJ e à Pro-Reitoria de Extensão Universitária da UFRJ pela concessão de bolsas (PROFAEX No 128/2017). Este trabalho recebeu apoio do Instituto Serrapilheira (número do processo Serra-1709-18891). 
1. HEALTH CANADA: Frequently Asked questions: "what is industrial hemp?".Disponível em: <http://www.hc-sc. gc.ca/hc-ps/substancontrol/hemp-chanvre/about-apropos/faq/index-eng.php\#a21> Acesso em 04/06/2017.

2. Bosy TZ, Cole KA. Consumption and quantitation of delta9-tetrahydrocannabinol in commercially available hemp seed oil products. J Anal Toxicol. 2000; 24(7):562566. DOI: $10.1093 /$ jat/24.7.562

3. Uchiyama N, Kikura-Hanajiri R, Kawahara N, Haishima Y, Goda Y. Identification of a cannabinoid analog as a new type of designer drug in a herbal product. Chem Pharm Bull 2009; 57(4): 439-441. DOI: 10.1007/s11419009-0069-y

4. Alves AO, Spaniol B, Linden R. Canabinoides sintéticos: drogas de abuso emergentes. Rev Psiquiatr Clín. 2012; 39(4): 142-148. DOI: 10.1590/S010160832012000400005

5. BRASIL. Ministério da Saúde. Portaria $n^{\circ} 344$, de 12 de maio de 1998. Aprovação do Regulamento Técnico sobre substâncias e medicamentos sujeitos a controle especial. Diário Oficial da União, Brasília. Secretaria de Vigilância Sanitária do Ministério da Saúde. 12 mai 1998.

6. BRASIL. Agência Nacional de Vigilância Sanitária. Resolução RDC nº 3, de 26 de janeiro de 2015. Disposição sobre a atualização do Anexo I, Listas de Substâncias Entorpecentes, Psicotrópicas, Precursoras e Outras sob Controle Especial, da Portaria SVS/MS no 344, de 12 de maio de 1998 e outras providências. Diário Oficial da União. 26 jan 2015.

7. BRASIL. Agência Nacional de Vigilância Sanitária. Resolução RDC n ${ }^{\circ} 17$, de 6 de maio de 2015. Definição dos critérios e procedimentos para a importação, em caráter de excepcionalidade, de produto à base de Canabidiol em associação com outros canabinóides, por pessoa física, para uso próprio, mediante prescrição de profissional legalmente habilitado, para tratamento de saúde. Diário Oficial da União. 17mai 2015.

8. Porter BE, Jacobson C. Report of a parent survey of cannabidiol-enriched cannabis use in pediatric treatment-resistant epilepsy. Epilepsy Behav. 2013; 29(3): 574-577. DOI: 10.1016/j.yebeh.2013.08.037.

9. Devinsky O, et al. Cannabidiol: Pharmacology and potential therapeutic role in epilepsy and other neuropsychiatric disorders. Epilepsia. 2014; 55(6):791-802. DOI: 10.1111/epi.12631.

10. Pedrazzi JFC, Pereira ACCI, Gomes FV, Bel ED. Perfil antipsicótico do canabidiol. Rev Medicina - Universidade de São Paulo. 2014; 47(2): 112-119.

11. Schier ARM et al. Canabidiol, um componente da Cannabis sativa, como um ansiolítico. Rev Bras Psiquiatr. 2012; 34(1). DOI: 10.1590/S151644462012000500008
12. Ivanovska V, Rademaker CMA, Dijk LV, Mantel-Teeuwisse AK. Pediatric Drug Formulations: A Review of Challenges and Progress. Pediatrics. 2014; 134(2). DOI: 10.1542/peds.2013-3225

13. Lopez FL, Ernest TB, Tuleu C, Gul MO. Formulation approaches to pediatric oral drug delivery: benefits and limitations of current platforms. Expert Opin Drug Deliv. 2015; 12(11) 1727-1740. DOI: 10.1517/17425247.2015.1060218.

14. Beauchamp GK, Mennella JA. Flavor Perception in Human Infants: Development and Functional Significance. Digestion. 2011; 83(1):1-6. DOI: 10.1159/000323397.

15. Mennella JA, Beuchamp GK. Optimizing Oral Medications for Children. Clin Ther. 2008;30(11): 2120-2132. DOI: $10.1016 /$ j.clinthera.2008.11.018

16. Mennella JA, Bobowski NK. The sweetness and bitterness of childhood: Insights from basic research on taste preferences. Physiol Behavior. 2015; 152(0): 502-507. DOI: 10.1016/j.physbeh.2015.05.015

17. Liem DG, Mennella JA. Sweet and Sour Preferences During Childhood: Role of Early Experiences. Dev Psychobiol. 2002; 41(4): 388-395. DOI: 10.1002/dev.10067

18. Mennella JA, Spector AC, Reed DR, Coldwell SE. The Bad Taste of Medicines: Overview of Basic Research on Bitter Taste. Clin Ther. 2013;35(8): 1225-1246. DOI: 10.1016/j.clinthera.2013.06.007

19. Mennella JA, Reed DR, Mathew PS, Roberts KM, Mansfield CJ. "A Spoonful of Sugar Helps the Medicine Go Down": Bitter Masking by Sucrose Among Children and Adults. Chem Senses.2015; 40(1): 17-25. DOI: 10.1093/ chemse/bju053.

20. Mennella JA, Finkbeiner S, Reed DR. The proof is in the pudding: children prefer lower fat but higher sugar than do mothers. Int J Obes. 2012; 36(10): 1285-1291. DOI: 10.1038/ijo.2012.51.

21. Liem DG, Mennella JA. Heightened Sour Preferences During Childhood. Chem Senses. 2003; 28(2): 173-180.

22. Mennella JA, Finkbeiner S, Lipchock SV, Hwang LD, Reed DR. Preferences for Salty and Sweet Tastes Are Elevated and Related to Each Other during Childhood. PLoS One. 2014;9(3): e92201. DOI: 10.1371/journal. pone.0092201

23. Ishizaka T, Okada S, Tokuyama E, Mukai J, Uchida T. Supression of Bitterness and Improvement of Palatability of Commercial Prednisolone Powder. Chem Pharm Bull. 2008; 56(10): 1395-1399.

24. Mintel's Global New Products Database. Flavor Insight 2012: Top Flavors in kids Candy. 2012[acesso 4 jun 2017]. Disponível em: https:/www.fona.com/sites/default/files/KidsCandy_FONAFlavorInsight_0812.pdf 
25. Mintel's Global New Products Database. Top Kids Flavors 2014: Sugar Confectionery. 2014[acesso 4 jun 2017]. Disponível em: http://info.symriseflavors.com/rs/ 549-NXL-800/images/KidsFlavorsSugarConfectionery. pdf

26. Heo, G.; Kim, S.H.; Chang, M.J. Effect of ketogenic diet and other dietary therapies on anti-epileptic drug concentrations in patients with epilepsy. J Clin Pharm Ther. 2017. DOI: $10.1111 /$ jept. 12578.

27. Hartman AL, Gasior M, MD, Vining EP, Rogawski MA. The Neuropharmacology of the Ketogenic Diet. Pediatr Neurol. 2007; 36(5): 281-292. DOI: 10.1016/j.pediatrneurol.2007.02.008

28. Beniczky S, Miranda MJ, Alving J, Heber Povlsen J, Wolf P. Effectiveness of the ketogenic diet in a broad range of seizure types and EEG features for severe childhood epilepsies. Acta Neurol Scand. 2000; 121(1):5862. DOI: 10.1111/j.1600-0404.2009.01303.x.

29. Hartman AL, Vinig EP. Clinical Aspects of the Ketogenic Diet. Epilepsia.2007; 48(1): 31-42. DOI: 10.1111/j. 1528-1167.2007.00914.x.

30. Monro JA, Shaw M. Glycemic impact, glycemic glucose equivalents, glycemic index, and glycemic load: definitions, distinctions, and implications. Am J Clin Nutr.2008; 87(1): 237S-243S.

31. BRASIL. Agência Nacional de Vigilância Sanitária. Resolução RDC n ${ }^{\circ} 18$, de 24 de março de 2008. Disposição sobre o "Regulamento Técnico que autoriza o uso de aditivos edulcorantes em alimentos, com seus respectivos limites máximos”. Diário Oficial da União. 24 mar 2008.

32. Grembecka M. Natural sweeteners in a human diet. Rocz Panstw Zakl Hig. 2015; 66(3): 195-202. DOI: PMID:26400114

33. ADA. American Dietetic Association. Position of the American Dietetic Association: use of nutritive and nonnutritive sweeteners. J Am Diet Assoc. 2004; 104(2).

34. Riera CE, Vogel H, Simon SA, le Coutre J. Artificial sweeteners and salts producing a metallic taste sensation activate TRPV1 receptors. Am J Physiol Regul Integr Comp Physiol. 2007; 293(2): 626-634. DOI: 10.1152/ajpregu. 00286.2007

35. Singla R, Jaitak V. Synthesis of rebaudioside A from stevioside and their interaction model with hTAS2R4 bitter taste receptor. Phytpchemistry.2016; 125: 106-111. DOI: 10.1016/j.phytochem.2016.03.004.
36. Ferreira AO, Oliveira A. Guia Prático da Farmácia Magistral. 2.ed. Juiz de Fora: Pharmabooks Editora; 2002.

37. Allen LV, Popovich NG, Ansel HC. Formas farmacêuticas e sistemas de liberação de fármacos. 9.ed. Porto Alegre: Artmed; 2013.

38. Singh Y, Bali C. Cannabis Extract Treatment for Terminal Acute Lymphoblastic Leukemia with a Philadelphia Chromosome Mutation. Case Rep in Oncol. 2013; 6(3): 585-592. DOI: 10.1159/000356446

39. Tzadok M, et al. CBD-enriched medical cannabis for intractable pediatric epilepsy. Seizure. 2016; 35: 41-44. DOI: $10.1016 /$ j.seizure.2016.01.004

40. Hussain SA, et al. Perceived efficacy of cannabidiol-enriched cannabis extracts for treatment of pediatric epilepsy: A potential role for infantile spasms and Lennox-Gastaut syndrome. Epilepsy Behav. 2015; 47: 138-141. DOI: 10.1016/j.yebeh.2015.04.009.

41. Leweke FM et al. Cannabidiol enhances anandamide signaling and alleviates psychotic symptoms of schizophrenia. Transl Psychiatry. 2012; 2(3): 94. DOI: 10.1038/ tp.2012.15.

42. Babu AS, Veluswamy SK, Arena R, Guazzi M, Lavie CJ. Virgin Coconut Oil and Its Potential Cardioprotective Effects. Postgrad Med. 2014; 126(7):76-83. DOI: 10.3810/ pgm.2014.11.2835.

43. Eyres L, Eyres MF, Chisholm A, Brown RC. Coconut oil consumption and cardiovascular risk factors in humans. Nutr Rev. 2016; 74(4):267-280. DOI: 10.1093/nutrit/ nuw002.

44. Huth PJ, Fulgoni VL, Larson BT. A Systematic Review of High-Oleic Vegetable Oil Substitutions for Other Fats and Oils on Cardiovascular Disease Risk Factors: Implications for Novel High-Oleic Soybean Oils. Adv Nutr. 2015; 6(6): 674-693. DOI: 10.3945/an.115.008979

45. Brasky TM et al. Specialty supplements and prostate cancer risk in the vitamins and lifestyle (VITAL) Cohort. Nutr Cancer. 2011; 63(4):573-582. DOI: 10.1080/01635581.2011.553022.

46. Nash DT. Cardiovascular risk beyond LDL-C levels. Other lipids are performers in cholesterol story. Postgrad Med. 2004; 116(3): 11-15.

47. Kalus U et al. Effect of Nigella sativa (black seed) on subjective feeling in patients with allergic diseases. Phytother Res. 2003; 17(10): 1209-1214. DOI:10.1002/ ptr. 1356 